\title{
Face and Face Parts Detection in Image Processing
}

\author{
Mirza Shahwar Haseeb, \\ Rana Muhammad Bilal Ayub, \\ *Muhammad Nadeem Ali \\ *Muhammad Adnan Khan
}

\begin{abstract}
This paper based on a procedure for automatically detecting one or more human faces, eyes pair, nose, mouth in colour images. This is depending on two-method which first detects regions of face, eyes pair, nose, mouth contain human skin in the colour image and then extracts information from these regions and then detect the regions which include face, eyes pair, mouth, nose in the colour image. The face, eyes pair, mouth, nose identified is completed on a colour image having only the identified image parts. An arrangement of thresh holding and calculated values and some functions are used to remove item structures that would show the existence of an identified area.
\end{abstract}

Keywords - vision.CascadeObjectDetector, Step, Rectangle.

\section{INTRODUCTION}

In recent years, it's most important and difficult how to control the security of information or systems. In recent years we know many law-breaking of computer, breakings by hackers, or security breaks in a business. In all these problems, the law-breaker were taking advantage of these systems and get access control in the system with the hack some information like ID cards, keys, passwords, PIN numbers. Now solution of these problem introduce a technology become giving confirmation of "true", separate [1]. This technology is also known as "bio-metrics". Biometric mean entrance control are automatic systems of verifying or identifying such as thumb print or facemask structures, or some features of the somebody's performance, similar his/her writing style or designs.

Face recognition is an example of biometric process that take the merits of both high correctness and low insensitivity. In newest years, face matching or its steps like (detection) has most search takings fast through not only computer science people but also scientist who need it, after this process it takes many expected uses in computer vision system and programmed access control system. Specially, face, eye, nose, mouth identifying is a first step of automatically face matching. But face, eye, nose, mouth identifying is not directly handle because it has many of differences of image points, such as difference face pose (front, non-front). ${ }^{2}$

The process for detection of faces, eyes pair, mouth, nose in this project was based on a two-step approach. First, the image is filtered or determine and checked is any parts of face or face in the image and, if available, return the image point and size of every faces or its parts and tell whether there is any human face or face parts, if there is, where it is (or where they are).

Mirza Shahwar Haseeb, Rana Muhammad Bilal Ayub,

*Muhammad Nadeem Ali and Muhammad Adnan Khan

Department of Computer Science, Lahore Garrison University

Lahore, Pakistan

Corresponding author email mnadeemali@lgu.edu.pk, 
Recognize and find people face in an image anyway of their point, scale, in-plane variation, angle, position (out-of-plane variation).

In this paper we presented a practical implementation of a frontal view face detection algorithm based on Viola-Jones approach using Matlab cascade object detector. Using the Matlab system object vision.CascadeObjectDetector, a face detector was developed configured to proceed the user organisation model specified in the input file. The file is made with the help of the vision.CascadeObjectDetector function. The attentional cascade training is done using a set of positive samples (windows with faces) and a set of negative images [2]. For obtaining a more accurate detector, the number of cascade layers and the function parameters were tuned. Finally, for different tuning parameters the performances of the face detector were analysed. This paper is prepared as follows. In Section II, presented Viola-Jones face detection algorithm. Section III illustrates the implementation of the Viola-Jones algorithm using Matlab cascade object detector. In section IV, we tested our proposal face detection system. Section V describes the conclusion and the future work.

\section{VIOLA - JONES ALGORITHM}

The Viola - Jones algorithm is introducing for real - time detection of faces, eye pair, nose, mouth from an image. Its real - time performance is obtained by using Haar type features, computed rapidly by using integral images, feature selection using the AdaBoost algorithm (Adaptive Boost) and faces, eye pair, nose, mouth detection with attentional cascade.

\subsection{Feature calculation}

Starting from the common characteristics of the faces, such as the area around the cheeks is whiter than the eyes or the area of the nose is brighter than those of the eyes, five Haar masks (Fig. 1)

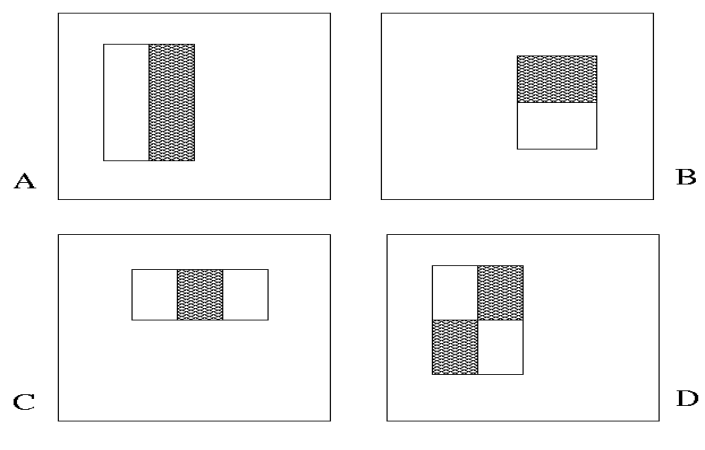

Figure 1 Five Haar Mask

Were chosen for determining the features, calculated at different positions and sizes. Haar features are calculated as the alteration between the addition of the pixels from the white area and the addition of the pixels from the dark area. In this way, it is possible to detect contrast differences. 


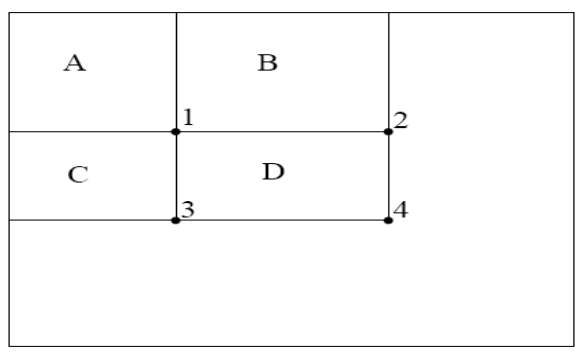

Figure 2 Detect Contrast

The addition of pixel in the $\mathrm{D}$ rectangle can be resolved by the four array positions like (A, B, C, $D)$. The addition of pixel rectangle $A$ at position 1 , the addition of pixel at position 2 is $A+B$, the addition of pixel at position 3 is $A+B+C$, the addition of pixel at position 4 is $A+B+C+D$. The value of $\mathrm{D}$ can be calculated as $4+1-(2+3)$.

\subsection{Attentional Cascade}

A strong classifier will result that classifies the windows of $N$ x $N$ size well enough. Since, on average, only $0.01 \%$ of the windows are positive images, meaning faces, only potentially positive windows must be examined.

Instead, to achieve a higher detection rate and a smaller misclassified images detection rate, we should use another strong classifier that classifies correctly the before misclassified images. This creates the attentional cascade, as showed in Fig. 4. At the first layer of the attentional cascade, a strong classifier with few features is used, which will filter/reject most negative windows [3]. A cascade of classifiers that are becoming more and more complex (with more features) will follow and they will allow to achieve a better detection rate. At each layer of the cascade, the negative images classified correctly will be eliminated and the new strong classifier will have a more difficult task than the previous step classifier.

Finally, the cascade of classifiers will operate as follows:

- the image will be split into multiple windows;

- every window is an input in the attentional cascade; -at every layer, the window is checked if it contains a face or not - according to the strong classifier;

- if it is negative, the window is rejected and the steps will be repeated for another window;

- if it is positive, it means that the window is a possible face and will move to the next layer of the cascade;

- The window contains a face if it passes all layers of the attentional cascade.

\section{DETECTOR IMPLEMENTATION USING MATLAB}

\subsection{Face Detection:}

For creating the system object that detects faces from an image, using the Viola - Jones algorithm, the following command is used:

FaceDectect $=$ vision. CascadeObjectDetector $($ 'FrontalFaceCART','MergeThreshold', 8);

After the creation of the detector, the step process is called by the following syntax:

FBOX = step (detector, I)The FBOX object return, an $\mathrm{M}-$ by -4 matrix describing $\mathrm{M}$ bounding boxes having the detected objects. Every row heaving 4 components [x y width height], that detect in pixels, the upper-left angle and size of a bounding box. 
In order to use a detector obtained from training, the following steps are done:

i) open the desired image;

ii) create the detector object;

iii) identify faces from the image;

iv)annotate the faces;

v) Show image with annotated faces.

\subsection{Eye Detection:}

For creating the system object that detects faces from an image, using the Viola - Jones algorithm, the following command is used:

EyeDectect = vision.CascadeObjectDetector ('EyePairBig','MergeThreshold', 8);

After the creation of the detector, the step process is called by the following syntax:

$\mathrm{EBOX}=$ step (detector, I)

The EBOX object return, an $\mathrm{M}-$ by -4 matrix describing $\mathrm{M}$ bounding boxes having the detected objects. Every row heaving 4 components [x y width height], that detect in pixels, the upper-left corner and size of a bounding box. In order to use a detector obtained from training, the following steps are done:
a. open the desired image;
b. create the detector object;
c. identify faces from the image;
d. annotate the faces;
e. Show image with annotated faces.

\subsection{Nose Detection:}

For creating the system object that detects faces from an image, using the Viola - Jones algorithm, the following command is used:

NoseDectect=vision.CascadeObjectDetector ('Nose','MergeThreshold', 20);

After the creation of the detector, the step method is called by the following syntax:

NBOX $=$ step (detector, I)

The NBOX object return, an $\mathrm{M}-$ by -4 matrix describing $\mathrm{M}$ bounding boxes having the detected objects. Every row heaving 4 components [x y width height], that detect in pixels, the upper-left corner and size of a bounding box. In order to use a detector obtained from training, the following steps are done:
a open the desired image;
b create the detector object;
c identify faces from the image;
d annotate the faces;
e Show image with annotated faces.

\subsection{Mouth Detection:}

For creating the system object that detects faces from an image, using the Viola - Jones algorithm, the following command is used:

MouthDectect=vision.CascadeObjectDetector ('Mouth','Merge Threshold', 95);

After the creation of the detector, the step method is called by the following syntax: 
MBOX = step (detector, I)

The MBOX object return, an $M-$ by -4 matrix describing $M$ bounding boxes having the detected objects. Every row heaving 4 components [x y width height], that detect in pixels, the upper-left corner and size of a bounding box.

In order to use a detector obtained from training, the following steps are done:
a open the desired image;
b create the detector object;
c identify faces from the image;
d annotate the faces;
e Show image with annotated faces.

\section{EXPERIMENTAL RESULTS}

The proposed face detection algorithm based on Viola-Jones was implemented using Matlab cascade object detector with different setting parameters of the Matlab function vision. CascadeObjectDetector, resulting many face, eye pair, nose, mouth detectors.

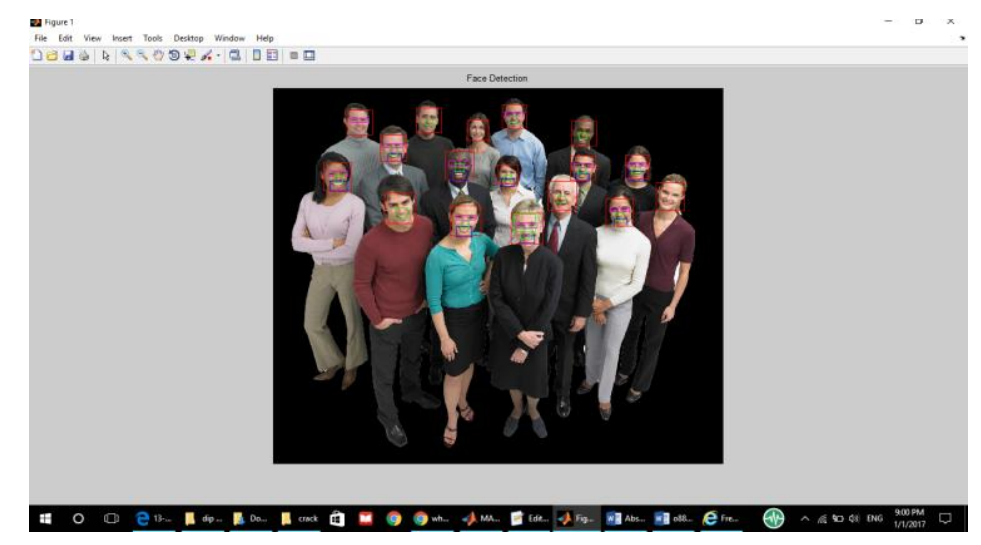

Figure 3 Results Face and Face Parts Detection

\section{CONCLUSIONS}

Using vision. Cascade Object Detector, a Matlab object system, a face, eye pair, nose, mouth detector based on Viola-Jones algorithm has been developed. Starting from several pertained classifiers for detecting frontal faces.

\section{REFERENCES}

[1]: S.-H. Lin, "An Introduction to Face Recognition Technology IC Media Corporation," IC Media Corporation, vol. 3, no. 3, pp. 1-7, 2000.

[2]: E. A. a. C. Lazar, "A Practical Implementation of Face Detection by Using Matlab Cascade Object Detector," in 19th International Conference on System Theory, 2015.

[3]: D. S. V. Ridhi Jindal Anuj Gupta, "Face Detection using Digital Image Proccessing," International Journal of Computer Sceince and Software Engineering, vol. 3, no. 11, November 2013. 